This item was submitted to Loughborough's Research Repository by the author.

Items in Figshare are protected by copyright, with all rights reserved, unless otherwise indicated.

\title{
Spin density wave selection in the one-dimensional Hubbard model
}

PLEASE CITE THE PUBLISHED VERSION

LICENCE

CC BY-NC-ND 4.0

\section{REPOSITORY RECORD}

Samson, J.H.. 2019. "Spin Density Wave Selection in the One-dimensional Hubbard Model". figshare. https://hdl.handle.net/2134/1209. 


\title{
Spin density wave selection in the one-dimensional Hubbard model
}

\author{
J H Samson \\ Department of Physics, Loughborough University of Technology, Loughborough, Leics \\ LE11 3TU, UK (Electronic address: j.h.samson@lut.ac.uk)
}

\begin{abstract}
The Hartree-Fock ground state phase diagram of the one-dimensional Hubbard model is calculated, constrained to uniform phases, which have no charge density modulation. The allowed solutions are saturated ferromagnetism (FM), a spiral spin density wave (SSDW) and a double spin density wave (DSDW). The DSDW phase comprises two canted interpenetrating antiferromagnetic sublattices. FM occurs for small filling, SSDW in most of the remainder of the phase diagram, and DSDW in a narrow tongue near quarter (and three-quarter) filling. Itinerant electrons lift the degeneracy with respect to canting angle in the DSDW. The Hartree-Fock states are metallic except at multiples of a quarter filling. Near half filling the uniform SSDW phase is unstable against phase separation into a half-filled antiferromagnetic phase and a hole-rich SSDW phase. The dependence of the ground state wave number on chemical potential is conjectured to be a staircase. Comparison is made with higher dimensional Hubbard models and the $J_{1}-J_{2}$ Heisenberg model.
\end{abstract}

PACS numbers: $75.10 \mathrm{Lp}, 75.25+\mathrm{z}$

cond-mat/9511116

To appear J. Phys.: Condens. Matter

Short title: Spin density waves in the 1D Hubbard model

March 7, 2006 


\section{Introduction}

The Hubbard Hamiltonian, originally proposed as a model of itinerant magnets, has lately gained new interest as a possible Hamiltonian for the cuprate superconductors. The Hamiltonian is

$$
H=H_{0}+U \sum_{i} n_{i \uparrow} n_{i \downarrow}
$$

where

$$
H_{0}=-\sum_{i j s} t_{i j} c_{i s}^{\dagger} c_{j s} .
$$

Here $i$ and $j$ are site indices, $s=\uparrow, \downarrow$ is a spin index and $U$ is the on-site Coulomb repulsion. We shall be considering the one-dimensional model with nearest-neighbour hopping, $t_{i j}=t$ for $|i-j|=1$ and $t=0$ otherwise, and arbitrary band filling $(0<n<2)$. One of the few exact results is the Bethe Ansatz ground state for this case (Lieb and Wu 1968). An approximate solution of the same model, especially one that breaks symmetries present in the exact solution, therefore requires some justification.

The aim of the present work is to investigate spin structures in the Hubbard model. Analogous questions arise in frustrated Heisenberg antiferromagnets, as reviewed by Schulz et al (1994). The simplest example is the one-dimensional Hamiltonian

$$
H=J_{1} \sum_{i} \mathbf{S}_{i} \cdot \mathbf{S}_{i+1}+J_{2} \sum_{i} \mathbf{S}_{i} \cdot \mathbf{S}_{i+2}
$$

with $J_{1}, J_{2}$ positive (Majumdar and Ghosh 1969). The classical ground state is a spiral; in the limit $J_{1} \ll J_{2}$, the even and odd sites decouple and the ground state becomes degenerate. In the spin $1 / 2$ case the peak in the structure factor moves from the Néel value $\pi$ to $\pi / 2$ with increasing $J_{2}$, as in the classical case (Tonegawa and Harada 1987, Zeng and Parkinson 1995). Unlike in the classical case, dimerization (local singlet formation) occurs for $J_{2} / J_{1}$ of the order unity or larger (Haldane 1982). The dimerization is not visible in two-spin correlation functions, but is seen in singlet-singlet correlations of the form $\left\langle\left(\mathbf{S}_{0} \cdot \mathbf{S}_{1}\right)\left(\mathbf{S}_{i} \cdot \mathbf{S}_{i+1}\right)\right\rangle$.

In certain situations the classical Heisenberg model possesses a line of degenerate ground states (in addition to the global rotational symmetry). The standard example in two dimensions is a square lattice antiferromagnet with nearest and next-nearest neighbour exchange interactions, with $J_{1}<2 J_{2}$. The classical ground state here comprises two interpenetrating antiferromagnetic sublattices, with no coupling between the Néel vectors. The three-dimensional face centred cubic antiferromagnet is similarly frustrated, with collinear spin density waves degenerate with a continuum of noncollinear double and triple spin density waves (see e.g. Long and Yeung 1986). These degeneracies could be lifted "by hand" by adding various terms to the classical 
Hamiltonian, for example anisotropy or biquadratic interactions. Such terms are not needed; "ordering by disorder" stabilizes the collinear phase, the disorder being either quantum (Shender 1982) or thermal (Henley 1987) in nature. On the other hand, Long (1989) and Henley (1989) have shown that non-magnetic impurities favour islands of the non-collinear phase localized about the impurity. Both effects are due to the large transverse susceptibility of each antiferromagnetic sublattice to fluctuations in the other sublattice. We will see here how an itinerant model provides another mechanism for state selection.

Similar spin structures arise in the Hubbard model; indeed, this reduces to the Heisenberg antiferromagnet in the half-filled two-sublattice large- $U$ limit. We shall be investigating Hartree-Fock (HF) solutions of the one-dimensional model. The paramagnetic HF state is always unstable for $U>0$ in one dimension. Perfect nesting ensures that the band susceptibility diverges at $q=2 k_{\mathrm{F}}$. The system is therefore unstable towards a spin density wave at this wave vector for infinitesimal $U$. For finite $U$ nonlinear effects can lead both to a shift in this wave vector and a distortion. We recognise that mean field theories in general, and HF calculations in particular, overestimate the tendency towards magnetic order (which the Mermin-Wagner theorem forbids in one dimension). However, the ordering may survive in the form of short-range correlations.

Most recent HF studies of the Hubbard model relate to the two-dimensional case, with reference to copper-oxygen planes in cuprate superconductors. An exhaustive search of solutions of the HF equations is impractical, with global minima hard to obtain and in any case dependent on the boundary conditions. Some authors restrict consideration to collinear magnetization, following Machida and Fujita (1984). These found an exact solution for a one-dimensional model with linearized dispersion: a soliton lattice with a snoidal spin density wave. Most studies find coplanar spin textures in the two-dimensional Hubbard model; however, Chubukov and Musaelian (1995) find evidence for non-coplanar textures. Vergés et al (1991) identify a large number of stable or metastable configurations. Spiral spin density waves (SSDW), with wave vectors varying continuously with $U$ and $n$ (Dzierzawa 1992), become unstable near half-filling. Here the HF ground state appears to be a collinear soliton lattice in which the holes are localized on walls between Néel-ordered domains (Fujita et al 1991, Ichimura et al 1992). The same conclusion follows from a fourth-order Landau expansion, valid for weak coupling, with coefficients determined from the electronic structure (Schulz 1990). Nearest-neighbour Coulomb repulsion tends to stabilize the SSDW phase against this hole clustering ( $\mathrm{Hu}$ et al 1994). One may identify various competing processes: firstly, a collinear spin density wave will present the electrons with a spatially varying potential, which may open a gap at the Fermi energy and stabilize the collinear wave. On the other hand, large $U$ might favour the more uniform charge density in a metallic 
SSDW. Competing ordered states, such as charge density waves and superconductivity, should also be taken into consideration (Bach et al 1994); however, for positive $U$ in the absence of other interactions the SSDW is favoured (Eriksson et al 1995). Some of the above features can already be seen in the one-dimensional case discussed in this work.

Early interest of course concentrated on the three-dimensional model (Penn 1966). There have been studies of spin structures in itinerant antiferromagnets, notably facecentred cubic $\gamma$-Mn, where single and multiple spin density waves are degenerate ground states of the classical Heisenberg model. Hirai and Jo (1985) show how fourth order terms in transfer integrals lift this degeneracy. However, spin density functional calculations reveal a very small energy difference between these structures (Crockford et al 1991). Here HF studies of the one-dimensional Hubbard model can illustrate the origin of the energy difference.

Bach et al (1994) have studied a generalized unrestricted HF theory, in which the quadratic HF Hamiltonian allows particle-nonconserving terms and therefore treats magnetic and BCS states on an equal footing. They report a number of theorems concerning the ground state symmetry, although not much is known rigorously about the finite- $U$ Hubbard model away from half filling.

The next section covers the solution of the HF equations. The solutions are restricted to uniform states, in which the local density of states is uniform up to spin rotation. Such states fall in two two-parameter families: spiral spin density waves (SSDW) and double spin density waves (DSDW). Each is parameterized by a field amplitude and the nearest-neighbour angle. The band structure is calculated for both families, and the energy is minimized with respect to the parameters. Section presents the resulting phase diagram: the DSDW is the ground state only in a narrow region near quarter (and three-quarter) filling. Near half filling, the homogeneous SSDW is unstable towards phase separation into a half-filled antiferromagnetic domain and a hole rich (or electron rich) domain. Finally, section 1 discusses the physical significance of the DSDW and the stability of the states found.

A brief report of some aspects of this work has recently appeared (Samson 1995).

\section{Computational method}

\subsection{The Hartree-Fock approximation}

The unrestricted HF approximation minimizes $\left\langle\left\{\boldsymbol{\Delta}_{i}, w_{i}\right\}|H|\left\{\boldsymbol{\Delta}_{i}, w_{i}\right\}\right\rangle$, the expectation value of the Hubbard Hamiltonian (11) in the space of Slater determinants $\left|\left\{\boldsymbol{\Delta}_{i}, w_{i}\right\}\right\rangle$. These states are ground states of the non-interacting many-electron system in a spinand site-dependent Hamiltonian

$$
H_{\mathrm{HF}}\left(\left\{\boldsymbol{\Delta}_{i}, w_{i}\right\}\right)=H_{0}+\sum_{i}\left(-\boldsymbol{\Delta}_{i} \cdot \mathbf{S}_{i}+w_{i} \cdot n_{i}\right)
$$


with

$$
\begin{aligned}
n_{i} & =\sum_{s} c_{i s}^{\dagger} c_{i s} \\
\mathbf{S}_{i} & =\frac{1}{2} \sum_{s t} c_{i s}^{\dagger} \sigma_{s t} c_{i t} .
\end{aligned}
$$

We take the number of sites $N_{\mathrm{a}} \rightarrow \infty$ and work in the canonical ensemble, with a fixed number $n$ of electrons per site. The general problem is a minimization of a function with a large number of local minima and saddle-points, dependent on boundary conditions and differing little in energy, in a $4 N_{\mathrm{a}}$-dimensional space. We shall therefore be far less ambitious and restrict consideration to uniform states, defined as those with a siteindependent spin-projected local density of states, referred to the local spin quantization direction. Defining the tight-binding Green function in the usual way as

$$
\left[G_{i j}\right]_{s t}(E)=\left\langle i s\left|\left(E-H_{\mathrm{HF}}\right)^{-1}\right| j t\right\rangle
$$

where $|i s\rangle$ is a one-electron Wannier orbital, we require the local tight-binding Green function to be of the form

$$
G_{i i}(E)=a(E)+b(E) \mathbf{e}_{i} \cdot \sigma .
$$

The only spatial dependence allowed is in the local magnetization directions, given by the unit vectors $\mathbf{e}_{i}$. All atoms are equivalent and there is no charge density modulation. The fields are then uniform in magnitude:

$$
w_{i}=\frac{1}{2} U n \text { and } \boldsymbol{\Delta}_{i}=\Delta \mathbf{e}_{i} .
$$

The energy then becomes

$$
E_{\mathrm{HF}}=\min _{\left\{\boldsymbol{\Delta}_{i}, w_{i}\right\}} V_{\mathrm{HF}}\left(\left\{\boldsymbol{\Delta}_{i}, w_{i}\right\}\right)
$$

where the minimization is subject to the constraints (9) and

$$
V_{\mathrm{HF}}\left(\left\{\boldsymbol{\Delta}_{i}, w_{i}\right\}\right)=\frac{1}{N_{\mathrm{a}}}\left\langle\left\{\boldsymbol{\Delta}_{i}, w_{i}\right\}\left|H_{\mathrm{HF}}\right|\left\{\boldsymbol{\Delta}_{i}, w_{i}\right\}\right\rangle+\frac{\Delta^{2}}{4 U}+\frac{1}{4} U n^{2} .
$$

This is equivalent to the self-consistency condition

$$
\boldsymbol{\Delta}_{i}=2 U\left\langle\left\{\boldsymbol{\Delta}_{i}, w_{i}\right\}\left|\mathbf{S}_{i}\right|\left\{\boldsymbol{\Delta}_{i}, w_{i}\right\}\right\rangle
$$

(so that in the HF solution the field is parallel to the magnetization).

\subsection{Spin density waves}

The uniformity condition (8) implies restrictions on the allowed directions, as we see from the expansion

$$
G_{i i}(E)=g_{i i}(E)-\frac{1}{2} \Delta \sum_{j} g_{i j}(E) \mathbf{e}_{j} \cdot \sigma g_{j i}(E)+\ldots
$$


where $g_{i j}(E)$ is the tight-binding Green function of the band Hamiltonian $H_{0}$ (2). Consistency between equations (13) and (8) for all energies requires the conditions

$$
\left(\mathbf{e}_{i-k}+\mathbf{e}_{i+k}\right) \| \mathbf{e}_{i} \quad \forall i, k .
$$

The configurations are therefore coplanar, which implies vanishing torque on the local moments (Small and Heine 1984). (Non-coplanar configurations are allowed in two or more spatial directions.) This allows only two classes of configuration, as shown in figure 11:

(i) Spiral spin density wave (SSDW)

$$
\mathbf{e}_{i}=(\sin Q i, 0, \cos Q i)
$$

(ii) Double spin density wave (DSDW)

$$
\begin{aligned}
& \mathbf{e}_{2 k}=(-1)^{k}(0,0,1) \\
& \mathbf{e}_{2 k+1}=(-1)^{k}(\sin \theta, 0, \cos \theta) .
\end{aligned}
$$

(We take the lattice parameter to be 1 , and the spins to be in the $x z$ plane.) The SSDW is specified by two parameters, the field amplitude $\Delta$ and the pitch angle $Q$, varying from 0 (the ferromagnetic phase $\uparrow \uparrow \uparrow \uparrow \ldots$ ) to $\pi$ (the antiferromagnetic phase $\uparrow \downarrow \uparrow \downarrow \ldots$ ). The DSDW is similarly specified by the amplitude $\Delta$ and an angle $\theta$. It may be thought of as two interpenetrating antiferromagnetic sublattices with the staggered magnetization vectors canted at an angle $\theta$, varying between 0 (the collinear or dimerized configuration $\uparrow \uparrow \downarrow \downarrow \ldots)$ and $\pi / 2$, which coincides with the $Q=\pi / 2$ SSDW $\uparrow \rightarrow \downarrow \leftarrow \ldots$ In the classical Heisenberg model the molecular field of one sublattice on the other vanishes in this phase, giving a two-dimensional manifold of degeneracies; any translationally invariant two-spin correlation function is independent of $\theta$. The DSDW is also a superposition of two SSDW states at $Q= \pm \pi / 2$.

\subsection{Band structures}

This restricted HF system is invariant under a subgroup of the symmetry group of the Hubbard Hamiltonian. Although translational and spin rotational symmetry are both broken, the Hamiltonian remains invariant under a simultaneous translation and spin rotation and Bloch's theorem still applies. We defer discussion of the stability of such configurations against further symmetry breaking to section 4.2 .

We calculate band structures of the HF Hamiltonian (4) in the standard way. Following Korenman et al (1977), we transform the spin quantization axis from the $z$ axis to the local axis $\mathbf{e}_{i}=\left(\sin \theta_{i}, 0, \cos \theta_{i}\right)$ :

$$
c_{i s}=\sum_{t} U_{s t}\left(\theta_{i}\right) d_{i t}
$$


where

$$
U(\theta)=\left(\begin{array}{cc}
\cos \theta / 2 & -\sin \theta / 2 \\
\sin \theta / 2 & \cos \theta / 2
\end{array}\right)
$$

The transfer integrals $t_{i j}$ couple the $\mathrm{d}_{\uparrow}^{\dagger}$ and $\mathrm{d}_{\downarrow}^{\dagger}$ bands, and the band structures reduce to the solution of second- and fourth-order secular equations for the SSDW and DSDW respectively. A little algebra leads to the two bands

$$
E_{\mathrm{SSDW}}(k)=-2 t \cos (Q / 2) \cos k \pm \sqrt{\Delta^{2} / 4+4 t^{2} \sin ^{2}(Q / 2) \sin ^{2} k}
$$

(with Brillouin zone $-\pi<k \leq \pi$ ) in the SSDW phase and the four bands

$$
E_{\mathrm{DSDW}}(k)= \pm \sqrt{\Delta^{2} / 4+2 t^{2} \pm \sqrt{\Delta^{2} t^{2}(1+\sin \theta \cos 2 k)+4 t^{4} \sin ^{2} 2 k}}
$$

(with Brillouin zone $-\pi / 2<k \leq \pi / 2$ ) in the DSDW phase.

Figure 2 shows sample band structures, illustrating how dimerization opens gaps in the folded ssDw bands. The Fermi surface follows from the condition that the length of $k$ space occupied is $2 \pi n$. For both families the Fermi surface always consists of either 0,2 or 4 points.

\subsection{Energy computation}

The energy of these configurations, following equation (11), is

$$
V_{\mathrm{HF}}(\Delta, Q \text { or } \theta)=\frac{1}{2 \pi} \sum_{\text {bands }}\left(\int_{E(k)<E_{\mathrm{F}}} E(k) d k\right)+\frac{\Delta^{2}}{4 U}+\frac{1}{4} U n^{2} .
$$

For the SSDW energies (19) this is evaluated in terms of the elliptic integral of the second kind, $E(\phi, k)$ (Gradshteyn and Ryzhik 1980):

$$
\begin{aligned}
& \int_{0}^{k_{\mathrm{F}}} E_{\mathrm{SSDW}}(k) d k=-2 t \cos (Q / 2) \sin k_{\mathrm{F}} \\
& \pm \frac{\Delta}{2}\left[\sqrt{1+p^{2}} E\left(\tan ^{-1}\left[\sqrt{1+p^{2}} \tan k_{\mathrm{F}}\right], p / \sqrt{1+p^{2}}\right)-\frac{p^{2} \sin k_{\mathrm{F}} \cos k_{\mathrm{F}}}{\sqrt{1+p^{2} \sin ^{2} k_{\mathrm{F}}}}\right]
\end{aligned}
$$

where

$$
p=(4 t / \Delta) \sin (Q / 2) \text {. }
$$

We evaluate the DSDW energies by numerical integration of the band structure $(20)$.

For each point $(n, U)$ in the phase diagram, we minimize the energies of the SSDW and DSDW states with respect to the parameters $(\Delta, Q)$ and $(\Delta, \theta)$ respectively. Because of perfect nesting, a non-zero sSDW solution always exists in some $Q$ interval for $U>0$. The DSDW phase requires a little more care, as the energy gain is small and a non-trivial solution is absent in much of the phase diagram. 


\section{Phase diagram}

\subsection{Energies}

Figure 3(a) shows the SSDW and DSDW HF energies for $U=4 t$ and $0 \leq n \leq 1$. Because of particle-hole symmetry, the range $1<n \leq 2$ contains no further information and will not be discussed further; references to quarter filling also apply to three-quarter filling. For comparison, the top curve in the figure shows the energy of the paramagnetic solution $(\Delta=0)$ and the bottom curve is Shiba's (1972) numerical solution of the Lieb-Wu integral equations for the exact ground state energy of the one-band one-dimensional Hubbard model. While the HF energies are not a good approximation to the exact ground state of this model, their relevance is discussed in section 4.2.

The SSDW ground state corresponds to saturated ferromagnetism for small $n$. The pitch $Q$ then increases continuously from 0 , reaching $\pi$ at half filling $(n=1)$. For small $U$, the nesting condition implies $Q \approx 2 k_{\mathrm{F}}=\pi n$; as $U$ increases, the ssDW moves to smaller $Q$, reducing double occupancy. The DSDW phase is stable with respect to the paramagnetic phase in a small range of fillings, and is only stable with respect to SSDW in a very narrow region near quarter filling $(n=1 / 2)$. The DSDW is most stable at exactly quarter filling, when the energy is minimized at the collinear DSDW $\theta=0$. The angle $\theta$ increases smoothly and monotonically (initially linearly) with deviation from quarter filling.

The above discussion applies for the range $0<U<U_{1} \approx 5.58 t$; for $U>U_{1}$ the DSDW state always has higher energy than the competing SSDW ground state (with $Q<\pi / 2$ ). Only for $U \approx 4 t$ can the DSDW region be seen in such a plot. It is difficult to find a DSDW solution numerically for small $U$. A fit in the range $t \leq U \leq 3 t$ suggests that the energy gain in forming a DSDW has the form $\exp \left(-U_{2} / U\right)$, with $U_{2} \approx 17 t$.

Suzumura and Tanemura (1995) have recently reported results on the HF ground state and excitations of the quarter-filled Hubbard model. They consider a different class of configurations, namely collinear $Q=\pi / 2$ spin density waves. Such states are pinned to the lattice by a small commensurability energy dependent on the phase of the wave. Their ground state, with phase $\pi / 4$, is precisely the DSDW (of energy $-0.681 t$ at

$U=4 t$, as calculated here). At all other phase angles, a $Q=\pi$ charge density wave coexists with the spin density wave. Maximum energy $(-0.679 t)$ occurs at zero phase, corresponding to a magnetic configuration $\uparrow \cdot \downarrow \cdot \ldots$. This is still less than our minimum SSDW energy of $-0.674 t$. They find the value of $U_{2} / t$ to be $4 \pi \sqrt{2} \approx 17.8$, compared with our numerical fit of 17 . 


\subsection{Phase diagram}

Figure $3(\mathrm{~b})$ shows the phase diagram in the $(n, U)$ plane. For $U>U_{\mathrm{c}}(n)$, indicated by the bold line, the ground state is saturated (strong) ferromagnetism (FM) with the upper band empty. The absence of unsaturated ferromagnetism is a consequence of the band-edge divergence of the one-dimensional density of states. The energy of this state (for $n \leq 1$ ) is independent of $U$, as there is no double occupancy:

$$
E_{\mathrm{HF}}=-\frac{2}{\pi} t \sin n \pi .
$$

The phase boundary (which appears to be linear for small $U$ ), can be calculated by expanding the band structure (19) to $O\left(Q^{2}\right)$ and integrating; the line where $\partial^{2} V_{\mathrm{HF}}(\Delta, Q) / \partial Q^{2}$ vanishes is

$$
U_{\mathrm{c}}(n)=\frac{2 \pi n-\sin 2 \pi n}{n \sin n \pi} .
$$

The limit $n \rightarrow 1$ gives $U_{\mathrm{c}} \rightarrow \infty$. Thus, in the infinite-repulsion limit, FM is (as expected) stable for all $n \neq 1$. At half filling $(n=1)$ the stable state is AFM. Figure 6 shows the energy of the uniform ground state (FM, SSDW or DSDW) as a function of $n$ for various values of $U$. Where the uniform ground state is FM, the energy coincides with the $U=\infty$ curve (24). DSDW is, as already mentioned, stable in only a small region of the phase diagram. All states are metallic apart from the AFM at half filling and the DSDW at quarter filling, where the Fermi level lies in a gap.

\subsection{Phase separation}

A curious feature of the SSDW energy is that it is not a convex function of $n$ near $n=1$. The uniform phase is therefore unstable to phase separation. Such an instability in the Hubbard model was proposed by Visscher (1974) and is analysed here by means of a Maxwell construction (Marder et al 1990, Arrigoni and Strinati 1991). The broken line $E_{\mathrm{ps}}(n)$ in figure $\mathrm{B}(\mathrm{a})$ coincides with $E_{\mathrm{HF}}(n)$ at $n=1$ and is tangent to the curve at $n=n_{1}(U)$, which is the boundary for phase separation. For $n_{1}<n<1$ the uniform SSDW is unstable towards a state with volume fraction $\left(n-n_{1}\right) /\left(1-n_{1}\right)$ of the $n=1$ AFM phase and $(1-n) /\left(1-n_{1}\right)$ of the $n=n_{1}$ SSDW phase. The energy of the phase-separated state is

$$
E_{\mathrm{ps}}(n)=\frac{\left(n-n_{1}\right) E_{\mathrm{HF}}(1)+(1-n) E_{\mathrm{HF}}\left(n_{1}\right)}{1-n_{1}}<E_{\mathrm{HF}}(n) .
$$

The boundary is shown as a broken line superposed on the phase diagram in figure $3(b)$. To the right of the boundary the uniform phase is unstable towards phase separation between the AFM phase and the FM or SSDW phase at the boundary. For $U>8 t$ (approximately), the only phases are FM and AFM. Andriotis et al (1993), in supercell 
calculations on the one-dimensional Hubbard model with collinear moments, see a phase separation of just this form for large $U$. In one dimension the phase separation is an artefact of the HF approximation, as the exact energy is a convex function of $n$ (Shiba 1972). If phase separation does occur in the Hubbard model in higher dimensions, it is a consequence of purely short-range interaction and would be suppressed by long-range Coulomb repulsion. On the other hand, we do not know whether the domains are of macroscopic size. The instability might signal phase separation at a more local level - the migration of holes to antiferromagnetic domain walls in a soliton lattice. This possibility depends on the sign of the domain wall energy.

Because of the kink in the energy at half filling there is no separation into $n>1$ and $n<1$ phases. A similar phase separation does occur between the SSDW and DSDW phases, implying that the pure DSDW phase is only stable at exactly quarter filling. To avoid complicating the diagram, this is omitted from figure 3 but is discussed in section 4.2 .

\section{Discussion}

\subsection{Interpretation of the $D S D W$}

The DSDW phase illustrates a number of physical phenomena. Firstly, it is an extreme case of a commensurate soliton lattice: an antiferromagnet with (uncharged) domain walls on alternate bonds. Secondly, the collinear DSDW is clearly stabilized near quarter filling by the opening of a gap at the Fermi energy (figure 2).

The HF energy can be expanded in the field:

$$
V_{\mathrm{HF}}\left(\Delta,\left\{\mathbf{e}_{i}\right\}\right)=V_{\mathrm{HF}}\left(0,\left\{\mathbf{e}_{i}\right\}\right)+\frac{1}{N_{\mathrm{a}}} \Delta^{2} \sum_{i k} J_{k} \mathbf{e}_{i} \cdot \mathbf{e}_{i+k}+O\left(\Delta^{4}\right) .
$$

The qualitative features of the phase diagram follow from a theorem concerning the dependence of susceptibilities on band filling (Heine and Samson 1980, 1983). Consider the moment expansion of the Green function

$$
G_{i j}(z)=\left[(z-H)^{-1}\right]_{i j}=\delta_{i j} z^{-1}+H_{i j} z^{-2}+\sum_{k} H_{i k} H_{k j} z^{-3}+\ldots
$$

where $H$ is a tight-binding Hamiltonian. The energy difference between two configurations A and B changes sign $r$ times as a function of band filling if the leading term in the difference of Green functions has the asymptotic form

$$
\operatorname{Tr} \sum_{i}\left[G_{i i}^{(\mathrm{A})}(z)-G_{i i}^{(\mathrm{B})}(z)\right] \sim z^{-r-3}
$$

for large $z$, where $\operatorname{Tr}$ is a trace over spin. A consequence is that the effective nearestneighbour exchange interaction $J_{1}$ changes sign twice in the band $0<n<2$, while $J_{2}$ changes sign four times. At quarter filling, $J_{1}$ is small and ferromagnetic and $J_{2}$ is larger 
and antiferromagnetic. Longer-range interactions are smaller. Hence the DSDW region of the phase diagram of the Hubbard model corresponds to the dimerized region of the Majumdar-Ghosh model discussed in the introduction.

The same theorem, applied to nonlinear susceptibilities, describes the state selection between collinear and noncollinear DSDW. The leading term in the $\theta$-dependence of the Green function must involve a biquadratic term in the angles. Leaving out the terms irrelevant to the present argument and summing equivalent paths gives

$$
\begin{aligned}
\operatorname{Tr} G_{00}^{\theta}(z) & =\ldots-\frac{1}{4} \operatorname{Tr}\left(\boldsymbol{\Delta}_{0} \cdot \sigma t_{01} \boldsymbol{\Delta}_{1} \cdot \sigma t_{10} \boldsymbol{\Delta}_{0} \cdot \sigma t_{01} \boldsymbol{\Delta}_{1} \cdot \sigma t_{10}\right) z^{-9}+\ldots \\
& =\ldots-\frac{1}{2} \Delta^{4} t^{4}\left[\left(\mathbf{e}_{0} \cdot \mathbf{e}_{1}\right)^{2}-\left|\mathbf{e}_{0} \times \mathbf{e}_{1}\right|^{2}\right] z^{-9}+\ldots \\
& =\ldots-\frac{1}{2} \Delta^{4} t^{4} \cos (2 \theta) z^{-9}+\ldots
\end{aligned}
$$

The energy difference between collinear and noncollinear DSDW states with equal $\Delta$ therefore has six zeros as a function of band filling (and a $\cos 2 \theta$ dependence). This agrees with the numerical results: the collinear phase is favoured near quarter, half and three-quarter filling, and the non-collinear phase at other values. Similar behaviour is indeed seen in calculations on $\gamma$-Mn (Long and Yeung 1986, 1987). The two-dimensional analogue seems to be the windmill configuration observed by Ichimura et al (1992) in a quarter filled square lattice. We note that the conclusion about the sign of the energy difference is not restricted to small $\Delta$, where a Landau expansion of the form (27) can be truncated at fourth order (Schulz 1990).

\subsection{Stability and relevance of solutions}

The solutions presented here (except at quarter and half filling) are metallic. In a onedimensional system we would still expect an instability towards a non-uniform state that opens a gap at the Fermi energy. Auerbach and Larson (1991) find just such an instability towards a local increase in the spiral pitch in the $t-J$ model. A similar instability is seen in the two-dimensional Hubbard model (Zhou and Schulz 1995). Phase separation is a further indication of this instability. If the filling is rational, the distortion would be commensurate; if it is irrational, an incommensurate distortion leading to a Cantor set spectrum is a possible outcome (Ostlund and Pandit 1984). Early studies of such spectra were more concerned with charge density waves, which, unlike the uniform SSDW, modulate the lattice potential. A similar modulation in the latter case requires a further distortion of the SSDW, leading to coexisting charge-density waves. This will result in a small kink in the $E(n)$ curves as in figure 6 . The true HF energy, according to the Maxwell construction, will be the convex hull of $E(n)$. The ground-state $Q$, plotted

as a function of chemical potential for constant $U$, would then be a staircase. The DSDW results and the small lock-in energies suggest that any energy gain will be small, and 
the tongues in the phase diagram will be correspondingly narrow. However, discussion of the fractal properties of this phase diagram will take us rather far from the physics of the Hubbard model.

The results reported here are consistent with known exact results for unrestricted generalized HF (Bach et al 1994). These authors discuss inter alia the HF ground state of the repulsive Hubbard model on a bipartite lattice. This is AFM for half filling, and FM in the $U \rightarrow \infty$ limit for all other fillings. They cannot obtain similar results in the interior of the phase diagram. However, the large gap and the work of Suzumura and Tanemura (1995) strongly suggest that the collinear DSDW is the true unrestricted HF ground state at quarter filling.

It must be admitted again that HF calculations fail to give the correct ground state symmetry, and give poor ground state energies; quantum fluctuations destroy AFM and SSDW order in one dimension, but may retain short-range correlations of that form. The higher-order correlations that distinguish the DSDW would be harder to see. Agreement appears to be better in two dimensions where HF solutions are a useful leading approximation (Mehlig 1993, Mehlig and Fulde 1994). For two and more dimensions the space to explore, even for uniform configurations, is much larger and an exhaustive study would be more time-consuming. On the other hand, symmetry breaking is possible in the ground state, so that HF calculations may give a ground state of the correct symmetry. The paramagnetic phase will be stable for small $U$ (except for the half-filled bipartite lattice), and the unrestricted HF energy $E(n)$ will be a smoother function than in one dimension, as it will be more difficult to open a gap. The calculations here can then be considered as a toy model, nevertheless giving physically relevant predictions of spiral phases and a special phase at quarter filling.

While the HF results give little information on the integrable one-band onedimensional model, the approximation is more useful in the degenerate case (which is not integrable). Many-electron atoms may be modelled by an $N$-band Hubbard Hamiltonian with inter-orbital Coulomb repulsion $(U / 4 N) n_{i}^{2}$ and Hund's rule term $-(I / N) S_{i}^{2}$, dependent on the total charge and spin on the atom. There is no reason that these should be governed by the same coupling constant for $N>1$. In the case $U \gg I$, Coulomb repulsion will suppress the phase-separated and non-uniform configurations, thereby stabilizing the SSDW against phase separation. The HF results for uniform states are independent of $N$, and are the leading approximation in the limit $N \rightarrow \infty$.

The energies obtained here may also be useful for finite temperature properties. In a study of the thermodynamics, Samson (1989) fitted the SSDW energies to an extended spherical model, obtaining indications that the correlation functions fall more slowly with temperature than in the Heisenberg model. That approximation however cannot distinguish the DSDW from the $Q=\pi / 2$ SSDW; the small energy gain suggests they would have little thermodynamic significance. 
While HF solutions of the one-dimensional Hubbard model clearly cannot give definitive answers to questions about higher dimensions, they do provide a model in which many of the physical processes underlying state selection operate in a transparent way.

\section{Acknowledgments}

The author is grateful to A S Alexandrov and M W Long for many helpful comments on the manuscript.

\section{References}

Andriotis A N, Economou E N and Soukoulis C M 1993 J. Phys.: Condens. Matter 5 4505-18

Arrigoni E and Strinati G C 1991 Phys. Rev. B 44 7455-65

Auerbach A and Larson B E 1991 Phys. Rev. B 43 7800-9

Bach V, Lieb E H and Solovej J P 1994 J. Statist. Phys 76 3-89

Chubukov A V and Musaelian K A 1995 Phys. Rev. B 51 12605-17

Crockford D J, Bird D M and Long M W 1991 J. Phys.: Condens. Matter 3 8665-82

Dzierzawa M 1992 Z. Phys. B 56 49-52

Eriksson A B, Einarsson T and Östlund S 1995 Phys. Rev. B 52 3662-75

Fujita M, Ichimura M and Nakao K 1991 J. Phys. Soc. Japan 60 2831-4

Gradshteyn I S and Ryzhik I M 1980 Table of Integrals, Series, and Products (London: Academic) formula 2.597 .2

Haldane F D M 1982 Phys. Rev. B 25 4925-8

Heine V and Samson J H 1980 J. Phys. F: Met. Phys. 10 2609-16

-1983 J. Phys. F: Met. Phys. 13 2155-68

Henley C L 1987 J. Appl. Phys. 61 3962-4 1989 Phys. Rev. Lett. 62 2056-9

Hirai K and Jo T 1985 J. Phys. Soc. Japan 54 3567-70

Hu F, Sarker S K and Jayaprakash C 1994 Phys. Rev. B 50 17901-9

Ichimura M, Fujita M and Nakao K 1992 J. Phys. Soc. Japan 61 2027-39

Korenman V, Murray J L and Prange R E 1977 Phys. Rev. B 16 4032-47

Lieb E H and Wu F Y 1968 Phys. Rev. Lett. 20 1445-8

Long M W 1989 J. Phys.: Condens. Matter 1 2857-74

Long M W and Yeung W 1986 J. Phys. F: Met. Phys. 16 769-90

1987 J. Phys. C: Solid State Phys. 20 5839-66

Machida K and Fujita M 1984 Phys. Rev. B 30 5284-99

Majumdar C K and Ghosh D K 1969 J. Math. Phys. 10 1388-98

Marder M, Papanicolaou N and Psaltakis G C 1990 Phys. Rev. B 41 6920-32

Mehlig B 1993 Phys. Rev. Lett. 702048

Mehlig B and Fulde P 1994 Z. Phys. B 94 335-9

Ostlund S and Pandit R 1984 Phys. Rev. B 29 1394-414

Penn D R 1966 Phys. Rev. 142 350-65 
Samson J H 1989 J. Phys.: Condens. Matter 1 6717-29

-1995 J. Magn. Magn. Mater. 140-144 205-6

Schulz H J 1990 Phys. Rev. Lett. 64 1445-8

Schulz H J, Ziman T A L and Poilblanc D 1994 in Magnetic Systems with Competing Interactions ed H T Diep (Singapore: World Scientific)

Shender E F 1982 Sov. Phys.-JETP 56 178-84 (translation of Zh. Exsp. Teor. Fiz. 83 326-37)

Shiba H 1972 Phys. Rev. B 6 930-8

Small L M and Heine V 1984 J. Phys. F: Met. Phys. 14 3041-52

Suzumura Y and Tanemura N 1995 J. Phys. Soc. Japan 64 2298-301

Tonegawa T and Harada I 1987 J. Phys. Soc. Japan 56 2153-67

Vergés J A, Louis E, Lomdahl P S, Guinea F and Bishop A R 1991 Phys. Rev. B43 6099-108

Visscher P B 1974 Phys. Rev. B 10 943-5

Zeng C and Parkinson J B 1995 Phys. Rev. B 51 11609-15

Zhou C and Schulz H J 1995 Phys. Rev. B 52 R11557-60 


\section{Figure captions}

Figure 1. Magnetization directions in (a) the SSDW and (b) the DSDW phases.

Figure 2. Band structures for (a) the SSDW with $\Delta=t$ and $Q=\pi / 2$ (equivalent to the DSDW with $\Delta=t$ and $\theta=\pi / 2$ ) and (b) the DSDW with $\Delta=t$ and $\theta=0$.

Figure 3. (a) HF results for the 1D Hubbard model as a function of band filling $n$ with $U=4 t$. The left-hand axis shows the pitch $Q$ of the SSDW (bold dashed line). The right-hand axis shows the energies. From top to bottom these are paramagnetic phase $(\Delta=0)$ (dash-dot line); SSDW phase (dotted); DSDW phase (full line); exact solution (bold line). The short dashed line on the right is the Maxwell construction. (b) The HF phase diagram for uniform phases (full lines). Between the dashed line and $n=1$ the uniform phase is unstable to phase separation.

Figure 4. Energy of the uniform HF ground state as function of band filling $n$ for various values of $U$. The $U=\infty$ curve corresponds to FM. 
arXiv:cond-mat/9511116 v1 23 Nov 1995

(q)

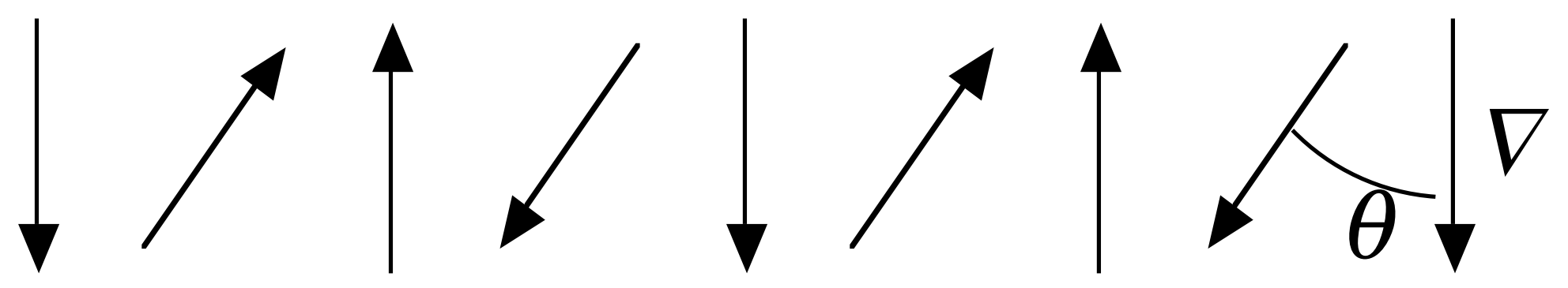

(e)

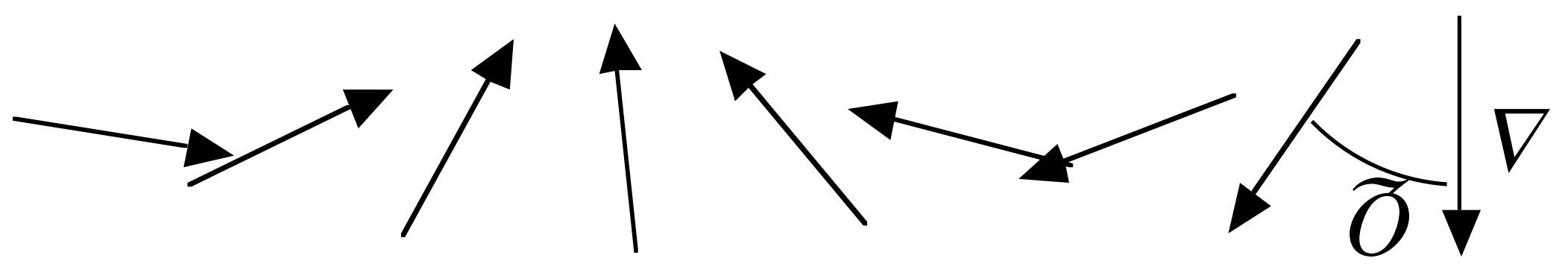




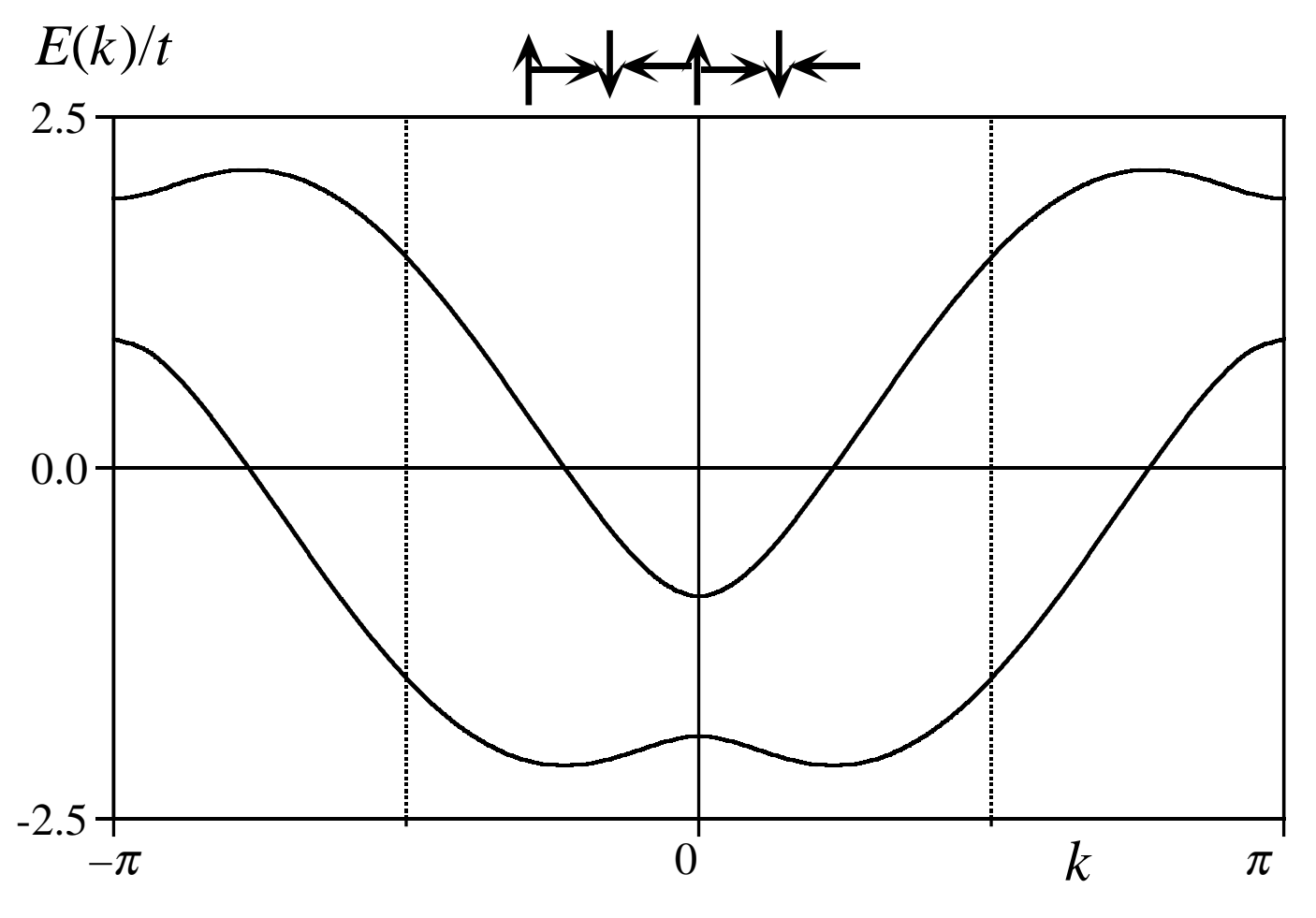

(a)

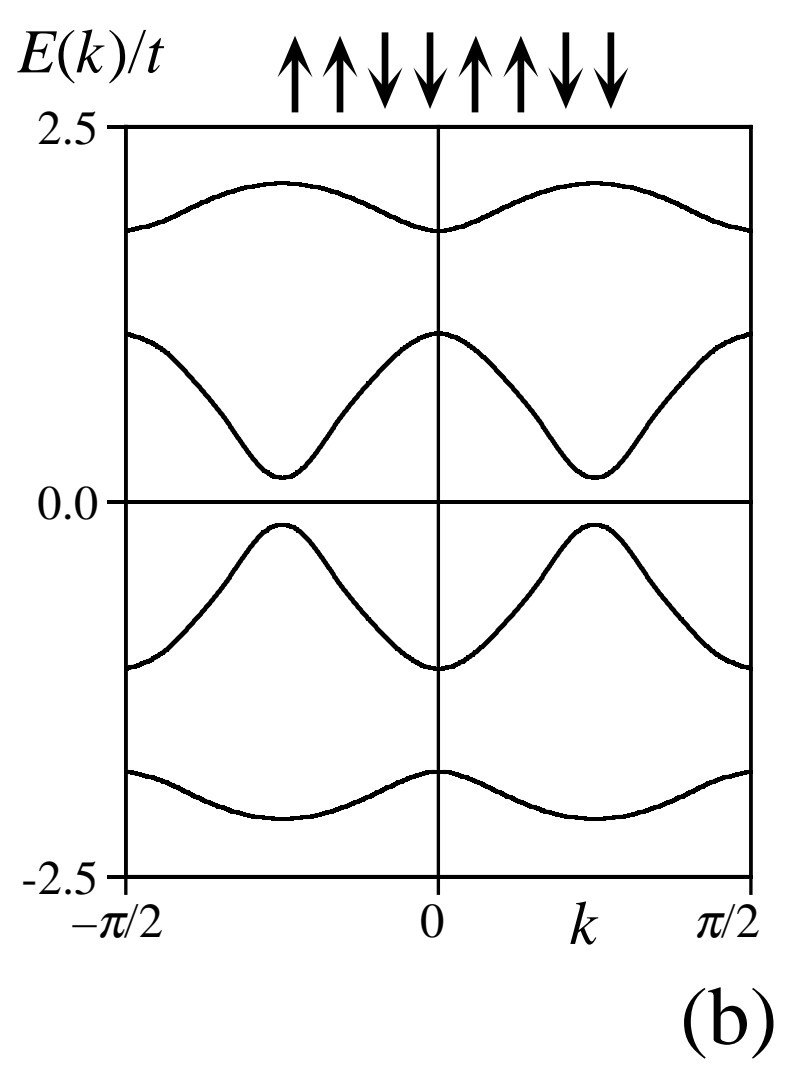




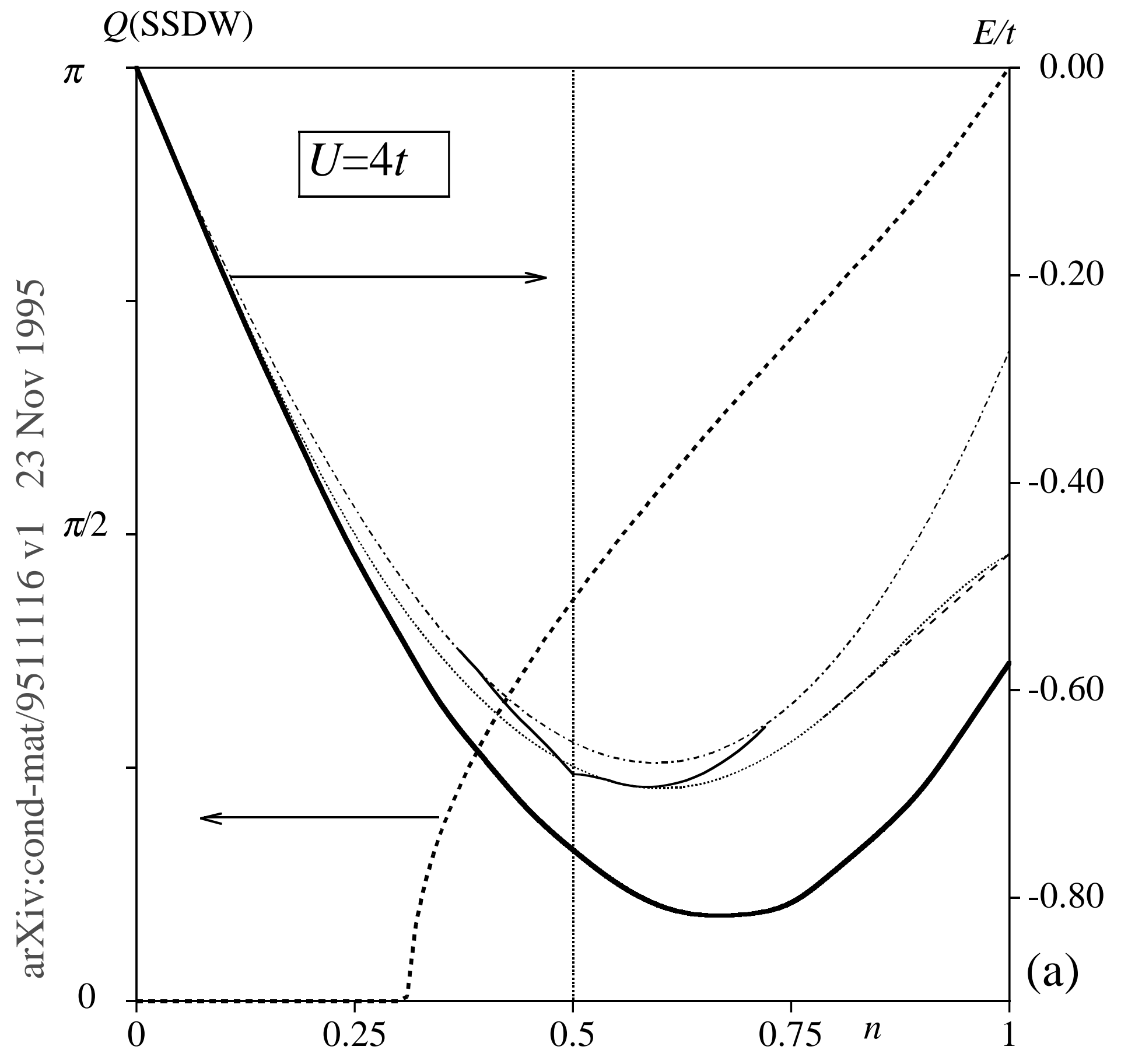


arXiv:cond-mat/9511116 v1 23 Nov 1995

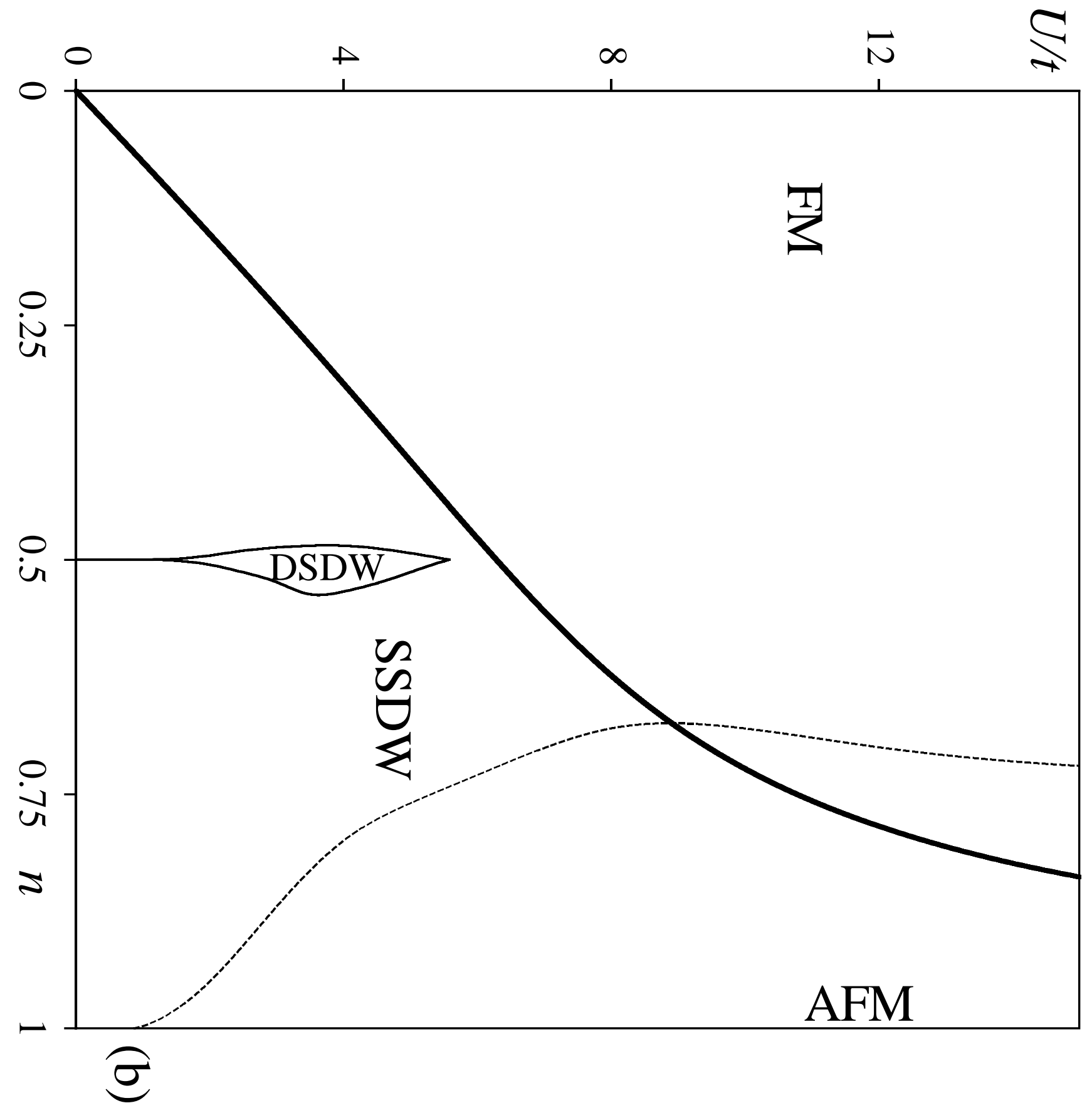




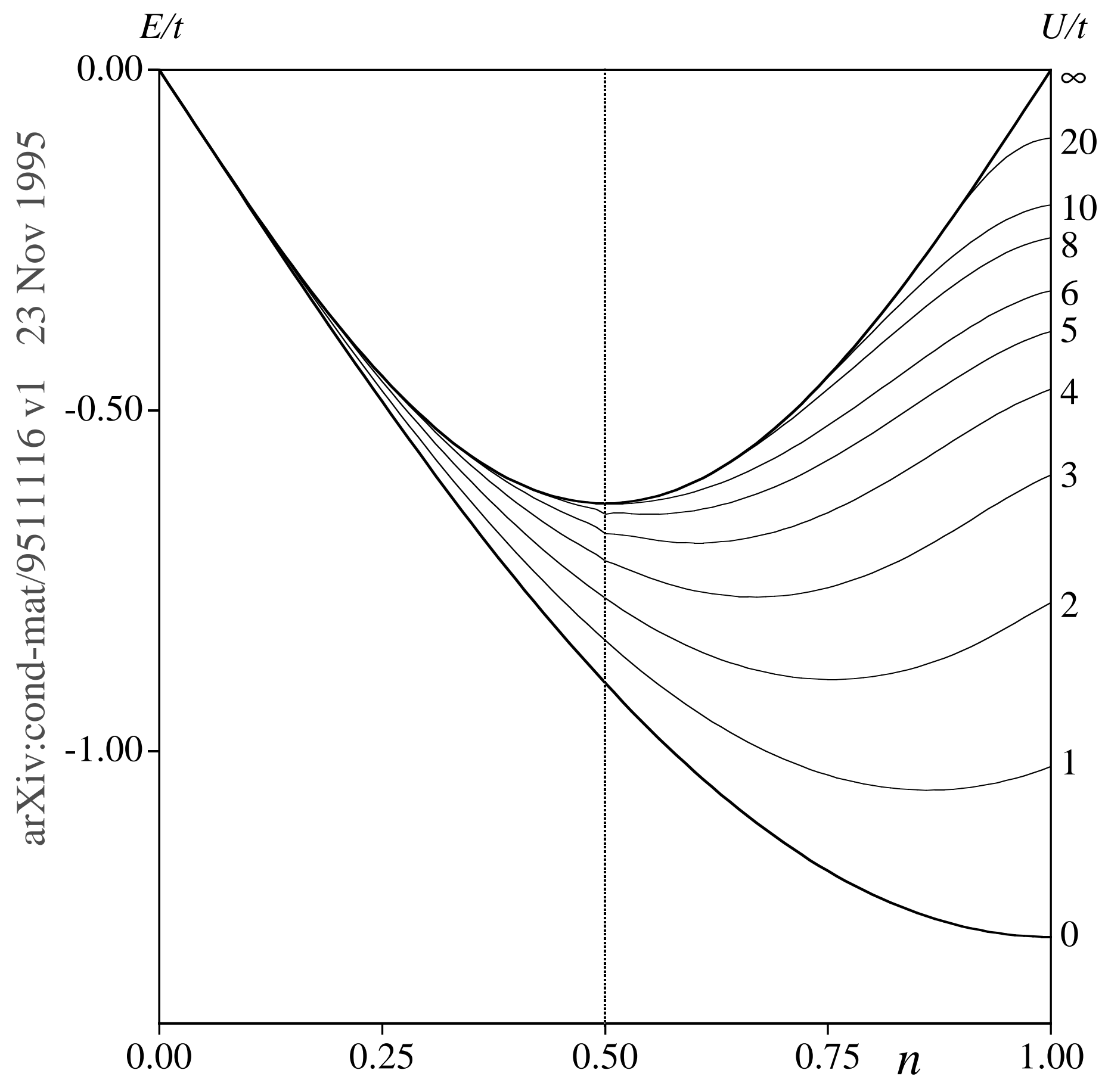

